\title{
MODAL AND TEMPORAL LOGICS FOR ABSTRACT SPACE-TIME STRUCTURES
}

\author{
SARA L. UCKELMAN, JOEL UCKELMAN
}

\begin{abstract}
In the 4th century BC, the Greek philosopher Diodoros Chronos gave a temporal definition of necessity. Because it connects modality and temporality, this definition is of interest to philosophers working within branching time or branching spacetime models. This definition of necessity can be formalized and treated within a logical framework. We give a survey of the several known modal and temporal logics of abstract space-time structures based on the real numbers and the integers, considering three different accessibility relations between spatio-temporal points.
\end{abstract}

\section{A Temporal Notion of Necessity}

Of the many different interpretations which can be given to the concept of necessity (logical necessity, physical necessity, deontic necessity, etc.), one of the most interesting from the formal point of view is the definition of necessity put forward by the Stoic logician Diodoros Chronos. Diodoros, a member of the Dialectical School who taught in Athens and Alexandria around 315-284 BC, is said to have defined the possible as that which either is or will be true, and the necessary as that which is true and will never be false. ${ }^{1}$ This definition of necessity was first analyzed formally by Prior, in Prior (1955) and Prior (1967), who provided an axiomatization of Diodorean necessity within the context of linear time structures. But it is within the context of branching time (or branching-space time) that Diodorean necessity has generated the most interest. This interest comes from two angles: The logicians who study abstract structures find the problem of axiomatizing the logics of time and necessity for branching time models an interesting theoretical question, and the philosophers who use branching time structures to model theories of agency and knowledge find the logical systems of these structures useful for their applicability to certain problems.

Key words and phrases. Diodoros Chronos, modal logic, necessity, $n$-dimensional space-time, temporal logic.

We would like to thank to participants in the Branching Space-Times Workshop, Kraków, October 24-25, 2005; the audience of the Logic Tea, Amsterdam, February 21, 2006; and the anonymous referees for their useful comments and suggestions.

${ }^{1}$ No writings of Diodoros remain; what we know of his position is second-hand coming from references by Epictetus, Cicero, and, indirectly, Boëthius. See Bobzien (2004) for more details. 
This paper takes the point of view of the theoretical logician interested in proving results about the logic of certain abstract mathematical structures, but with an aim at making these theories accessible to the philosopher who may wish to use these logics for practical purpose. So while the philosopher may be most interested in the four-dimensional structure of Minkowski space-time, as it is the basis of Einstein's theory of relativity and hence has practical application in reality, we discuss here branching structures of arbitrary dimension, even though these may not have any counterpart in reality.

We begin with a brief introduction to the logics which we will use for analyzing these structures, before turning to the structures themselves. Our basic logic will be the propositional calculus, that is, arbitrary propositions combined with the Boolean operators $\neg, \wedge, \vee$, and $\rightarrow$, where all propositional tautologies are taken as axioms.

For each structure we consider both the modal logic and the temporal logic of that structure. For this we need both a modal language and a temporal language:

Definition 1.1. The modal language $\mathcal{L}(\square)$ is standard propositional logic language $\mathcal{L}$ to which we add an operator $\square$, which is read 'necessarily'. We read $\square^{n} p$ as $\underbrace{\square \ldots \square}_{\mathrm{n} \text { times }} p$.

The type of necessity expressed by the operator $\square$ is simple logical necessity, that is, the only necessities are the propositional tautologies.

Definition 1.2. The temporal language $\mathcal{L}(G, H)$ is standard propositional logic language $\mathcal{L}$ to which we add two operators $G$ 'necessarily in the future' and $H$ 'necessarily in the past'.

In each language, we define the duals of these operators as follows:

$$
\begin{aligned}
\diamond & =\neg \square \neg \\
F & =\neg G \neg \\
P & =\neg H \neg \\
\diamond^{n} & =\neg \square^{n} \neg
\end{aligned}
$$

The first three operators are read 'possibly', 'possibly in the future', and 'possibly in the past', respectively.

We represent our structures as forms of Kripke models. A Kripke model $M$ is a tuple $\langle W, R, V\rangle$, where $W$ is a set of points (which can be thought of as 'worlds' or 'times'), $R$ is a binary relation on $W$, and $V$ is a function from propositional variables to $\wp(W) . V(p)$ is understood to be the set of worlds or times when the proposition $p$ is true. Truth in a model is defined recursively in the expected manner for the Boolean 
connectives, with the following clauses for the new operators being:

$$
\begin{array}{llll}
M, x \vDash \square \phi & \text { iff } & \forall y x R y & M, y \vDash \phi \\
M, x \vDash \square^{n} \phi & \text { iff } & \forall y x R^{n} y & M, y \vDash \phi \\
M, x \vDash G \phi & \text { iff } & \forall y x R y & M, y \vDash \phi \\
M, x \vDash H \phi & \text { iff } & \forall y y R x & M, y \vDash \phi
\end{array}
$$

When we place no constraint on the type of necessity expressed by $\square$, the modal logic that results is minimal. It is called $\mathrm{K}$, and it consists of all axioms of propositional logic plus the single modal axiom

$$
\square(p \rightarrow q) \rightarrow(\square p \rightarrow \square q)
$$

The rules of inference are modus ponens, uniform substitution, and necessitation (from $\vdash \phi$ conclude $\vdash \square \phi$ ).

When no constraints are placed on the nature of time, the temporal logic that results is minimal. It is called $\mathrm{K}_{\mathrm{t}}$, and it contains all axioms of propositional logic plus the axioms

$$
\begin{aligned}
H(p \rightarrow q) & \rightarrow(H p \rightarrow H q) \\
G(p \rightarrow q) & \rightarrow(G p \rightarrow G q) \\
p & \rightarrow H F p \\
p & \rightarrow G P p
\end{aligned}
$$

The first two axioms are just the temporal analogs of the modal axiom, and the second two axiomatize the fact that the $H$ and $G$ operators are converses of each other. As with $\mathrm{K}, \mathrm{K}_{\mathrm{t}}$ has as rules of inference modus ponens and uniform substitution, plus necessitation axioms for each operator (from $\vdash \phi$ conclude $\vdash H \phi$ and $\vdash G \phi$ ).

If we wish to use a Diodorean-type necessity with its connection between time and necessity, we can do so in two ways. First, we can work solely within the modal language, and place a constraint on the models that we use. This constraint is that the relation $R$ must be reflexive, and forces, by the truth conditions, that whenever $p$ is necessary at a point, it is also true at that point, and also that whenever $p$ is true at a point, it is possible at that point. Second, we can work solely within the temporal language, and introduce $\square p$ 'necessarily $p$ ' as a definition for $p \wedge G p$ ' $p$ and $p$ is always true in the future'.

\section{An Abstract Characterization of the Structures}

As noted above, the structures that we consider are arbitrary $n$ dimensional structures based on either the real numbers $(\mathbb{R})$ or the integers $(\mathbb{Z})$. We present the known results and open problems concerning modal and temporal axiomatizations of such structures. While questions about non-modal or temporal logics describing these structures are interesting, they are beyond the scope of this paper and we say nothing about them here. 
In structures with dimension $n$, the members of $W$ are named by $n$ tuples. There are three different binary relations on $n$-tuples in these structures that are of interest. Models where $W=\mathbb{R}^{n}$ were first investigated because of their connections with relativistic $n$-dimensional spacetime. For example, $\mathbb{R}^{4}$ represents Minkowski spacetime when the relation $R$ is $\preceq$, defined as follows:

$$
\bar{a} \preceq \bar{b} \text { iff } \sum_{i=1}^{n-1}\left(b_{i}-a_{i}\right)^{2} \leq\left(b_{n}-a_{n}\right)^{2} \text { and } a_{n} \leq b_{n}
$$

Intuitively, the set $\{y: x \preceq y\}$ is the future light-cone of $x$, that is, the set of all space-time points which are accessible from $x$ and hence are part of $x$ 's future. Events which occur outside of this future light-cone cannot be affected by $x$.

$\left\langle\mathbb{R}^{n}, \preceq\right\rangle$ is isomorphic to $\left\langle\mathbb{R}^{n}, \leq\right\rangle$; the second is just the rotation of the first by $45^{\circ}$. Because they are isomorphic, results which can be proved about one of the structures apply to the other as well. As a result, we may work solely with $\left\langle\mathbb{R}^{n}, \leq\right\rangle$ as is easier to visualize.

It is clear that $\preceq$ as defined is a reflexive order (and hence one that would be suitable to use when considering the Diodorean-type necessity discussed earlier). There are two irreflexive orders which are natural to consider. The first is $\prec$, which is the irreflexive version of $\preceq$ above:

$$
\bar{a} \prec \bar{b} \text { iff } \sum_{i=1}^{n-1}\left(b_{i}-a_{i}\right)^{2}<\left(b_{n}-a_{n}\right)^{2} \text { and } a_{n}<b_{n}
$$

The second relation is the 'after' relation introduced in Robb's (1914) seminal work on time:

$$
\bar{a} \alpha \bar{b} \text { iff } \exists k a_{k}<b_{k} \text { and } \forall i \neq k a_{i} \leq b_{i}
$$

It is not intuitively clear how $\prec$ differs from $\alpha$; the difference is best illustrated by comparing pictures of the 2-dimensional cases, Figure 1 and Figure 2.

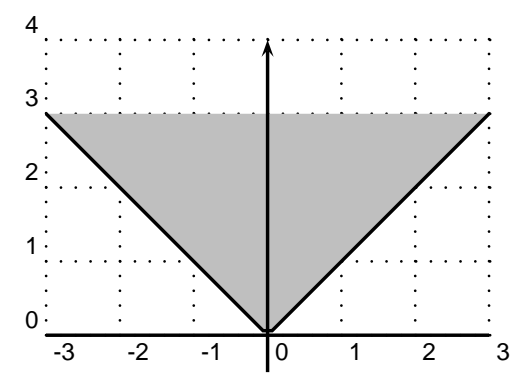

Figure 1: $\left\langle\mathbb{R}^{2}, \alpha\right\rangle$

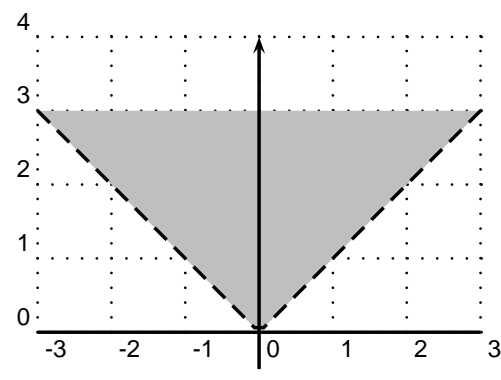

Figure $2:\left\langle\mathbb{R}^{2}, \prec\right\rangle$

$\langle 0,0\rangle \prec\langle 1,1\rangle$ iff $(1-0)^{2}<(1-0)^{2}$, which is not the case. Hence, $\langle 0,0\rangle \nprec\langle 1,1\rangle$, but $\langle 0,0\rangle \alpha\langle 1,1\rangle$. 
Note that unlike the case for $\preceq$, rotation of $\prec$ by $45^{\circ}$ does not result in an isomorphic structure. The difference which is being captured in these two irreflexive orderings is that the future $\alpha$-lightcone of a tuple of points $\bar{x}$ contains all tuples which can be reached from $\bar{x}$ traveling at speeds up to and include the speed of light, whereas the future $\prec-$ lightcone of the same tuple contains all and only the tuples which can be reached traveling at less than the speed of light.

Though we are restricting our attention to just $\mathbb{R}^{n}, \mathbb{Z}^{n}, \preceq, \prec$, and $\alpha$, it should be clear that this still leaves us with a very wide range of possible structures about which we may inquire of their modal and temporal logics. We turn now to doing precisely this.

\section{Results for Structures $\mathbb{R}^{n}$}

As noted above, structures of $\mathbb{R}^{n}$ are of interest because of their use in modelling relativistic space-time. These structures were first investigated in Goldblatt (1993), and he proved the initial results in this area.

Theorem 3.1. The Diodorean modal logic of the frames $\left\langle\mathbb{R}^{n}, \leq\right\rangle$ for all $n \geq 2$ is S4.2. S4.2 is K plus the following axioms:

$$
\begin{aligned}
\mathbf{4} & =\square p \rightarrow \square \square p \\
\mathbf{T} & =\square p \rightarrow p \\
\mathbf{g a} & =\diamond \square p \rightarrow \square \diamond p
\end{aligned}
$$

This logic is finitely axiomatizable, complete, and has the finite model property.

Proof. Goldblatt (1993).

These three additional axioms each correspond with a different property of the structure. Axiom 4 corresponds to the transitivity of the relation $\leq$. Axiom $\mathbf{T}$ corresponds to the reflexivity of the relation. Axiom ga corresponds to a property called connectedness (also called the diamond property, or Church-Rosser), which states that if $\bar{a} \leq \bar{b}$ and $\bar{a} \leq \bar{c}$, then there is a $\bar{d}$ such that $\bar{b} \leq \bar{d}$ and $\bar{c} \leq \bar{d}^{2}$

Theorem 3.2. The modal logics for the frames $\left\langle\mathbb{R}^{n}, \prec\right\rangle$ for $n \geq 2$ is $\mathrm{L}_{2} . \mathrm{L}_{2}$ is $\mathrm{K}$ plus the following axioms:

$$
\begin{aligned}
\mathbf{4} & =\diamond \diamond p \rightarrow \diamond p \\
\mathbf{D} & =\diamond(p \vee \neg p) \\
\mathbf{D}_{\mathbf{2}} & =(\diamond p \wedge \diamond q) \rightarrow \diamond(\diamond p \wedge \diamond q) \\
\mathbf{g a} & =\diamond \square p \rightarrow \square \diamond p
\end{aligned}
$$

\footnotetext{
${ }^{2}$ For a general discussion of correspondences between logical axioms and properties of a structure's relation, see Chagrov \& Zakharyaschev (1997), chapters 3 and 4 .
} 
Proof. Shapirovsky \& Shehtman (2003).

Axiom $\mathbf{D}$ corresponds to the property of seriality, that is, there are no dead ends, every tuple can access another tuple. Axiom $\mathbf{D}_{\mathbf{2}}$ corresponds to a property called 2-density, which is the property:

$$
\forall \bar{x} \forall \bar{y}_{1} \forall \bar{y}_{2} \exists \bar{z}\left(\left(\bar{x} \prec \bar{y}_{1} \wedge \bar{x} \prec \bar{y}_{2}\right) \rightarrow\left(\bar{x} \prec \bar{z} \wedge \bar{z} \prec \bar{y}_{1} \wedge \bar{z} \prec \bar{y}_{2}\right)\right)
$$

The fact that the modal logics of the reflexive and irreflexive versions of the frames $\mathbb{R}^{n}$ are the same no matter what the dimension is (so long as it's greater than 1) is quite unique: This is is not the case with known results about any of the relations on $\mathbb{Z}^{n}$.

We end our discussion of the $\mathbb{R}^{n}$ structures by noting that no axiomatization has yet been given for either the modal or the temporal $\operatorname{logics}$ of $\left\langle\mathbb{R}^{n}, \alpha\right\rangle$, for any $n$. There is only this result:

Theorem 3.3. The temporal logics for $\left\langle\mathbb{R}^{n}, \alpha\right\rangle$ are distinct for every $n$.

Proof. Corollary 3.2 of Phillips (1998). ${ }^{3}$

\section{Results for Structures $\mathbb{Z}^{n}$}

When we move to considering $\mathbb{Z}^{n}$ instead of $\mathbb{R}^{n}$, the situation becomes much more complicated. Whereas the modal logic for $\left\langle\mathbb{R}^{n}, \leq\right\rangle$ is the same no matter which $n \geq 2$ you pick, this is not the case with $\left\langle\mathbb{Z}^{n}, \leq\right\rangle$.

Theorem 4.1. The modal logics for each $\left\langle\mathbb{Z}^{n}, \leq\right\rangle$ are all distinct.

Proof. Phillips (1998). This follows from the fact that there is a formula which is true on the $n$-dimensional frame which is false on the $n+1$ dimensional frame. We fix $n$. First, define the following abbreviations:

$$
\mathbf{A L L}=\bigwedge_{1 \leq i \leq n+1} p_{i} \quad \mathrm{NONE}=\bigwedge_{1 \leq i \leq n+1} \neg p_{i} * \mathbf{p}_{\mathbf{i}}=\left(p_{i} \wedge \bigwedge_{\substack{1 \leq j \leq n+1 \\ j \neq i}} \neg p_{j}\right)
$$

\footnotetext{
${ }^{3}$ This corollary has a confusing typo: The corollary claims that the modal logics of each $\left\langle\mathbb{R}^{n}, \alpha\right\rangle$ are all distinct. However, it is clear that this was not the intended statement of the corollary, since Phillips claims that it follow directly from the previous theorem, which discusses the temporal logics of $\left\langle\mathbb{Z}^{n}, \alpha\right\rangle$.
} 
Then, the following formula schema is valid on $\left\langle\mathbb{Z}^{n}, \leq\right\rangle$, and invalid on $\left\langle\mathbb{Z}^{n+1}, \leq\right\rangle$ :

$$
\begin{gathered}
\left(N O N E \wedge \square\left(N O N E \rightarrow \square\left(A L L \vee N O N E \vee \bigvee_{1 \leq i \leq n+1} * p_{i}\right)\right) \wedge\right. \\
\square\left(N O N E \rightarrow \bigwedge_{1 \leq i \leq n+1} \diamond * p_{i}\right) \wedge \\
\left.\bigwedge_{\substack{1 \leq i \leq n+1 \\
1 \leq i, j \leq n+1 \\
i \neq j}} \nabla\left(p_{i} \rightarrow \square\left(* p_{i} \vee A L L\right)\right)\right) \rightarrow
\end{gathered}
$$

This proof (and the formula within it) is essentially just an application of the pigeonhole principle: At $n$ dimensions, there simply isn't enough space to make the various combinations of $n+1$ propositions in the antecedent true, but when you step up to $n+1$ dimensions, there is such space.

Theorem 4.2. The temporal logics for each $\left\langle\mathbb{Z}^{n}, \alpha\right\rangle$ are all distinct.

Proof. Phillips (1998). This follows from the fact that there is a formula which is true on the $n$-dimensional frame which is false on the $n+1$ dimensional frame. We fix $n$. First, define the following abbreviation:

$$
\mathrm{Q}_{\mathbf{i}}=p_{i} \rightarrow \bigwedge_{\substack{1 \leq j \leq n+1 \\ j \neq i}} \neg p_{j} \wedge G \neg p_{j}
$$

Then, the following formula schema is valid on $\left\langle\mathbb{Z}^{n}, \alpha\right\rangle$, and invalid on $\left\langle\mathbb{Z}^{n+1}, \alpha\right\rangle$ :

$$
\bigwedge_{1 \leq i \leq n+1} F p_{i} \wedge G Q_{i} \rightarrow \bigvee_{1 \leq i<j \leq n+1} F\left(F p_{i} \wedge F p_{j}\right)
$$

This formula works in a fashion similar to the one in the previous theorem.

Corollary 4.3. The modal logics for each $\left\langle\mathbb{Z}^{n}, \alpha\right\rangle$ are all distinct.

Proof. This follows from Theorem 4.2. The temporal formula used to distinguish the dimensions uses only the forward-looking operators $F$ and $G$ operators, which have exactly the same truth conditions as $\diamond$ and $\square$. Hence, using definitions analogous to the ones above (replacing Gs with $\square \mathrm{s})$, the following formula schema is valid on $\left\langle\mathbb{Z}^{n}, \alpha\right\rangle$ and invalid on $\left\langle\mathbb{Z}^{n+1}, \alpha\right\rangle$ :

$$
\bigwedge_{1 \leq i \leq n+1} \diamond p_{i} \wedge \square Q_{i} \rightarrow \bigvee_{1 \leq i<j \leq n+1} \diamond\left(\diamond p_{i} \wedge \diamond p_{j}\right)
$$




\section{Open Problems and Concluding Remarks}

The proofs and axiomatizations given above are far from being a complete story. There is still much work that remains to be done in completely characterizing the modal and temporal logics for these classes of abstract structures. We highlight here some of the interesting open questions which remain to be solved, as well as some conjectures concerning the answers:

Problem 5.1. What is an axiomatization of the temporal logic of $\left\langle\mathbb{R}^{n}, \leq\right\rangle$ ?

The answer to this question is currently not known, but it is our conjecture that it is simply the temporal analog of the modal logic:

Conjecture. The axiomatization of the temporal logic of $\left\langle\mathbb{R}^{n}, \leq\right\rangle$ is $K_{t}$ plus

$$
\begin{aligned}
\mathbf{4}_{\mathbf{t}} & =G p \rightarrow G G p \\
\mathbf{4}_{\mathbf{t}} & =H p \rightarrow H H p \\
\mathbf{T}_{\mathbf{t}} & =G p \rightarrow p \\
\mathbf{T}_{\mathbf{t}} & =H p \rightarrow p \\
\mathbf{g a}_{\mathbf{t}} & =F G p \rightarrow G F p \\
\mathbf{g a}_{\mathbf{t}} & =P H p \rightarrow H P p
\end{aligned}
$$

However, no proof of this conjecture has yet been forthcoming.

Problem 5.2. What is an axiomatization of the temporal logic of $\left\langle\mathbb{R}^{n}, \prec\right\rangle$ ?

Conjecture. Phillips (1998) conjectures that it is the same for each $n$, but beyond that, no further information is yet known.

Problem 5.3. What is an axiomatization of the temporal logic of $\left\langle\mathbb{Z}^{n}, \leq\right\rangle$ ?

Problem 5.4. What is an axiomatization of the modal and temporal logics of $\left\langle\mathbb{Z}^{n}, \prec\right\rangle$ ?

Conjecture. Phillips (1998) conjectures that the logics are the same for each $n$, but then notes that Byrd (unpublished) has shown that the frames $\left\langle\mathbb{Z}^{n}, \prec\right\rangle$ are in fact distinct, though does not elaborate.

The known results discussed in $\S \S 3,4$ fall into two types: structures where the logics are identical even when the dimension of the structure varies, and structures where the logics vary in a uniform fashion when 


\begin{tabular}{|c|c|c|c|}
\hline \multicolumn{2}{|c|}{ Structure } & Modal Logic & Temporal Logic \\
\hline \hline \multirow{3}{*}{$\mathbb{R}^{n}$} & $\preceq / \leq$ & for $n \geq 2$, all identical & open \\
\cline { 2 - 4 } & $\prec$ & for $n \geq 2$, all identical & open \\
\cline { 2 - 4 } & $\alpha$ & open & for $n \geq 2$, each $n$ unique \\
\hline \hline \multirow{3}{*}{$\mathbb{Z}^{n}$} & $\preceq / \leq$ & for $n \geq 2$, each $n$ unique & open \\
\cline { 2 - 4 } & $\prec$ & open & open \\
\cline { 2 - 4 } & $\alpha$ & for $n \geq 2$, each $n$ unique & for $n \geq 2$, each $n$ unique \\
\hline
\end{tabular}

Table 1: Summary of Results

the dimension of the structure varies. We present a summary of these results, plus the open problems in Table 1 .

From this summary of the current state of affairs, we would not be surprised to find all of the open slots in the table for $\mathbb{Z}^{n}$ eventually filled in with proofs of uniqueness of the logics for each dimension, nor would we be surprised to find that the temporal logics for $\left\langle\mathbb{R}^{n}, \preceq\right\rangle$ and $\left\langle\mathbb{R}^{n}, \prec\right\rangle$ are the same no matter what $n$ is. It is our hope that some of these gaps will be filled in the near future.

\section{REFERENCES}

[1] Bobzien, S. (2004). "Dialectical school", in Zalta, E.N., The Stanford encyclopedia of philosophy, Fall 2004 Edition, http://plato.stanford.edu/archives/ fall2004/entries/dialectical-school/

[2] Chagrov, A. \& Zakharyaschev, M. (1997). Modal logic. Oxford: Clarendon Press.

[3] Goldblatt, R. (1992). Logics of time and computation, 2nd. ed. Stanford: CSLI Publications.

[4] Goldblatt, R. (1993). "Diodorean modality in Minkowski spacetime", Mathematics for modality. Stanford: CSLI Publications, ch. 8. Originally in Studia Logica 39 (1980), 219-236.

[5] Phillips, J.F. (1998). "A note on the modal and temporal logics for $n$ dimensional spacetime", Notre Dame Journal of Formal Logic 39(4), 545-553.

[6] Phillips, J.F. (1999). Modal logics for integral spacetime. (Doctoral dissertation, University of Wisconsin-Madison, 1999).

[7] Phillips, J.F. (2001). "Modal logics of succession for 2-dimensional integral spacetime" Journal of Philosophical Logic 30, 1-25.

[8] Prior, A.N. (1955). "Diodoran modalities", Philosophical Quarterly 5(20), 205213.

[9] Prior, A.N. (1967). Past, present, and future. Oxford: Oxford University Press.

[10] Robb, A.A. (1914). A theory of time and space. Cambridge: University Press.

[11] Shapirovsky, I. \& Shehtman, V. (2003). "Chronological future modality in Minkowski spacetime", Advances in modal logic, 4, 437-459.

Institute for Logic, Language, and Computation, Universiteit van Amsterdam, Plantage Muidergracht 24, 1018 TV Amsterdam, The NetherLANDS

E-mail address: suckelma@illc.uva.nl, juckelma@illc.uva.nl 